\author{
Marjolein H. Liedenbaum \\ Ayso H. de Vries \\ Steve Halligan \\ Patrick M. M. Bossuyt \\ Abraham H. Dachman \\ Evelien Dekker \\ Jasper Florie \\ Stefaan S. Gryspeerdt \\ Sebastiaan Jensch \\ C. Daniel Johnson \\ Andrea Laghi \\ Stuart A. Taylor \\ Jaap Stoker
}

Received: 16 October 2008

Revised: 23 December 2008

Accepted: 6 January 2009

Published online: 18 February 2009

(C) The Author(s) 2009.

This article is published with open access at

Springerlink.com

M. H. Liedenbaum $(\bowtie)$.

A. H. de Vries - P. M. M. Bossuyt .

E. Dekker · J. Florie $\cdot$ J. Stoker

Department of Radiology,

Academic Medical Center,

Meibergdreef 9,

1105 AZ, Amsterdam, Netherlands

e-mail: m.h.liedenbaum@amc.uva.nl

Tel.: +31-20-5663657

Fax: +31-20-5669119

S. Halligan

University College Hospital,

London, UK

A. H. Dachman

The University of Chicago Medical

Center,

Chicago, IL, USA

\title{
CT colonography polyp matching: differences between experienced readers
}

S. S. Gryspeerdt · S. A. Taylor

Stedelijk Ziekenhuis,

Roeselare, Belgium

S. Jensch

Onze Lieve Vrouwe Gasthuis,

Amsterdam, Netherlands

\section{D. Johnson}

Mayo Clinic Scottsdale,

Scottsdale, AZ, USA

A. Laghi

University of Rome "Sapienza",

Latina, Italy
Abstract The purpose of this study was to investigate if experienced readers differ when matching polyps shown by both CT colonography (CTC) and optical colonoscopy (OC) and to explore the reasons for dis- crepancy. Twenty-eight CTC cases with corresponding $\mathrm{OC}$ were pre- sented to eight experienced CTC readers. Cases represented a broad spectrum of findings, not completely fulfilling typical matching criteria. In 21 cases there was a single polyp on

$\mathrm{CTC}$ and $\mathrm{OC}$; in seven there were multiple polyps. Agreement between readers for matching was analyzed. For the 21 single-polyp cases, the number of correct matches per reader varied from 13 to 19. Almost complete agreement between readers was observed in 15 cases $(71 \%)$, but substantial discrepancy was found for the remaining six $(29 \%)$ probably due to large perceived differences in polyp size between $\mathrm{CT}$ and OC. Readers were able to match between $27(71 \%)$ and $35(92 \%)$ of the 38 CTC detected polyps in the seven cases with multiple polyps. Experienced CTC readers agree to a considerable extent when matching polyps between CTC and subsequent $\mathrm{OC}$, but non-negligible disagreement exists.

Keywords CT colonography • Colorectal neoplasia $\cdot$ Colorectal polyps · Polyp matching . Colonoscopy

\section{Introduction}

Computed tomography colonography (CTC) is an established diagnostic technique for both symptomatic and screening patients [1]. The diagnostic performance of CTC has generally necessitated comparison between CTC and subsequent optical colonoscopy (OC) [2]. Such comparisons are often performed by an experienced radiologist who uses prespecified criteria to match polyps identified by $\mathrm{CTC}$ with those found at subsequent $\mathrm{OC}[2,3]$.
A variety of matching criteria have been described, usually based on the location, size, and morphology of polyps [4-8]. For example, a correct matching may be assumed when the polyp identified by CTC is found in the same or adjacent colonic segment as the polyp detected by OC. For convenience the colon is usually divided into six segments: caecum, ascending, transverse and descending colon, sigmoid and rectum [2,3]. For size matching a frequently described criterion stipulates that the CTC polyp must be within $50 \%$ of the diameter measured at colonos- 
copy. Matching based on general morphology may also be performed.

However, observers using identical criteria may nevertheless match different polyps because the matching procedure is subjective and requires interpretation of both CTC and colonoscopy data. Furthermore, variation in the matching criteria stipulated by different researchers hinders comparisons between different studies.

The purpose of our study was to investigate to what extent experienced readers differ when matching polyps between CTC and $\mathrm{OC}$ and to explore the reasons underpinning any differences. Ultimately we aimed to develop criteria to minimize matching disagreement with a view to improve study methodology and facilitate interstudy comparisons.

\section{Methods}

Eight highly experienced CTC researchers from six centers in Europe and the USA participated. We administered a questionnaire to document their current criteria for polyp matching. To investigate matching in daily practice, the eight readers were asked to match preselected cases. Reader experience varied from 250 to over 3,000 CTC interpretations and between 100 and 3,000 CTC cases with corresponding OC.

\section{Questionnaire}

The questionnaire presented multiple choice questions relating to matching criteria used by readers for their research studies (Table 1). Options were formulated based on descriptions of matching criteria from the literature [4-8].

\section{Patients}

A radiology researcher (M. L.) selected 28 cases from two research databases of 170 surveillance patients and 240 fecal occult blood test positive patients who had undergone both CTC and subsequent colonoscopy. These studies had been approved by the local Medical Ethics Committee and informed consent was obtained from all participants.

All CTC examinations had been read prospectively by one of four experienced observers, each with at least 100 CTC interpretations with colonoscopic verification. Observers had marked any polyp and indicated the morphology, size, location, and their confidence.

Colonoscopy with segmental unblinding was performed subsequently by a gastroenterologist, gastroenterology resident, or gastroenterology nurse under supervision. Maximal polyp diameter was estimated by using an opened biopsy forceps and in some cases with a linear measure probe additionally (Olympus America). All colonoscopies were videotaped starting from the caecum.

\section{Case selection}

As the present study aimed at evaluating concordance when matching polyps between CTC and OC, selection was biased towards cases likely to prove challenging. A research fellow experienced in matching ( $>250$ matched CTC and OC studies) selected cases with polyps that failed to meet the typical matching criteria specified by the literature [4-8]. For example, a polyp at CTC whose location apparently differed by two segments or more from the location suggested by colonoscopy. Only technically adequate CTC examinations were selected so as not to confound matching, by insufficient distension, for example.

Twenty-one cases were selected where a single polyp at CTC and OC had to be matched by the experienced reader. To evaluate a broad spectrum of potential matching scenarios, in 13 cases the CTC and colonoscopy data were purposely perturbed so that two different patients were combined. In this way, different morphologies and/or locations could be presented to the reader. Figure 1 illustrates examples of three cases. Seven other cases were selected that had multiple polyps at CTC and/or OC. Again, difficult cases were purposely selected whose polyps could not necessarily be matched using established criteria.

\section{Reviewing matching cases}

All readers performed the observations at their own department and were free from clinical commitments during the matching procedure. Readers were free to use their own visualization software to read cases, but a laptop with View Forum software (Version 6.2, Philips, Best, Netherlands) was also available. Polyps initially found by the CTC observer (in the original research study) were presented to the readers by a researcher with information on morphology, size, location, and certainty of diagnosis scored by the observer. The experienced readers were able to remeasure the polyps if they wished. Colonoscopic information was also available to the readers: colonoscopy videos, diameter information, and location and morphology.

\section{Polyp matching}

Readers completed a data form for each case. For the 21 single-polyp cases the readers indicated whether they considered the CTC and OC polyp a correct match. If readers believed the two polyps were not the same, the researcher queried their reasoning and classified each mismatch as due to disagreement relating to: (1) diameter; (2) morphology; (3) location.

In the seven multiple-polyp cases, readers were invited to indicate which of the polyps presented to them matched and which they believed did not. Again, reasons for mismatching were explored. 
Table 1 Questionnaire matching

1. What information do you use for the matching procedure? (more than one answer possible)

$\square$ Colonoscopy video

$\square$ Colonoscopy report

$\square$ Pictures of colonoscopy polyps

$\square$ Other:.

2. What information from the gastroenterologist concerning the polyps you need for matching? (more than one answer possible)

$\square$ Size of the polyp

$\square$ Segmental location

$\square$ Morphology

$\square$ Distance of endoscope from anus to lesion

$\square$ Information of pathologist about histology

$\square$ Other:

3. Do you use morphology criteria for matching CTC polyps with colonoscopy polyps?

$\square$ Yes $\rightarrow$ go to question 4

$\square$ No $\rightarrow$ go to question 5

4. If yes, morphology matching is based on (more than one answer possible):

$\square$ The description of the morphology of the gastroenterologist (flat, sessile, pedunculated) resembles the description of the morphology of the CTC observer.

$\square$ The polyp at CTC has a similar appearance/shape as the colonoscopy polyp (judged by the observer that is performing the matching)

$\square$ Other:.

5. What is the definition you use for flat lesions? *(Please fill in the number of $\mathrm{mm}$ )

$\square$ The width is at least 2 times the height of the lesion

$\square$ The lesion protrudes less than .. $\mathrm{mm}^{*}$ from the mucosa

$\square$ The surface of the lesion is flat and not convex

$\square$ Other:

6. Do you use size criteria for matching a CTC polyp with a colonoscopy polyp?

$\square$ Yes, the CTC polyp and colonoscopy polyp have to be within a certain size range

$\square$ Yes, the CTC polyp and colonoscopy polyp need to have the same size

$\square$ No, size is no criterion for matching $\rightarrow$ go to question 8

7. If matching is done according to size range, the size range must be within: (* indicate percentage) $\square$ The CTC polyp must be maximally...\%* smaller and ....\%* larger than the colonoscopy polyp $\square$ The colonoscopy polyp must be maximally...\%* smaller or ....\%* larger than the CTC polyp $\square$ The size of the lesion at CTC can be maximally ....\%* smaller than the measured lesion size at colonoscopy or can be any size larger

$\square$ Other:

8. Do you use the segmental location for matching the CTC and colonoscopy polyps?

$\square$ Yes $\rightarrow$ go to question 9

$\square$ No

9. If yes, the location matching is based on:

$\square$ The CTC polyp must be in the same segment (e.g. cecum, ascending, transverse or descending colon, sigmoid or rectum) as the colonoscopy polyp

$\square$ The CTC polyp must be in the same or most nearby half of the adjacent segment as the colonoscopy polyp

$\square$ The CTC polyp must be in the same or adjacent segment as the colonoscopy polyp

$\square$ The CTC polyp must be in the same or next 2 adjacent segments as the colonoscopy polyp

$\square$ The CTC polyp must be in the same or next ... adjacent* segments as the colonoscopy polyp (*please indicate number)

$\square$ Other: 
Fig. 1 a Case 2; CTC polyp: caecum, $7.1 \mathrm{~mm}$, sessile. OC polyp: ascending colon, $3 \mathrm{~mm}$, sessile. From left to right: 2D image, 3D image, colonoscopy image. In this case all eight readers indicated a match. b Case 15; CTC polyp: sigmoid, $5.2 \mathrm{~mm}$, sessile. OC polyp: ascending colon, $6 \mathrm{~mm}$, sessile. In this case only one reader indicated a match. c Case 19; CTC polyp: descending colon, $17.9 \mathrm{~mm}$, pedunculated. OC polyp: pedunculated, $6 \mathrm{~mm}$, pedunculated. Four of the eight readers indicated a match
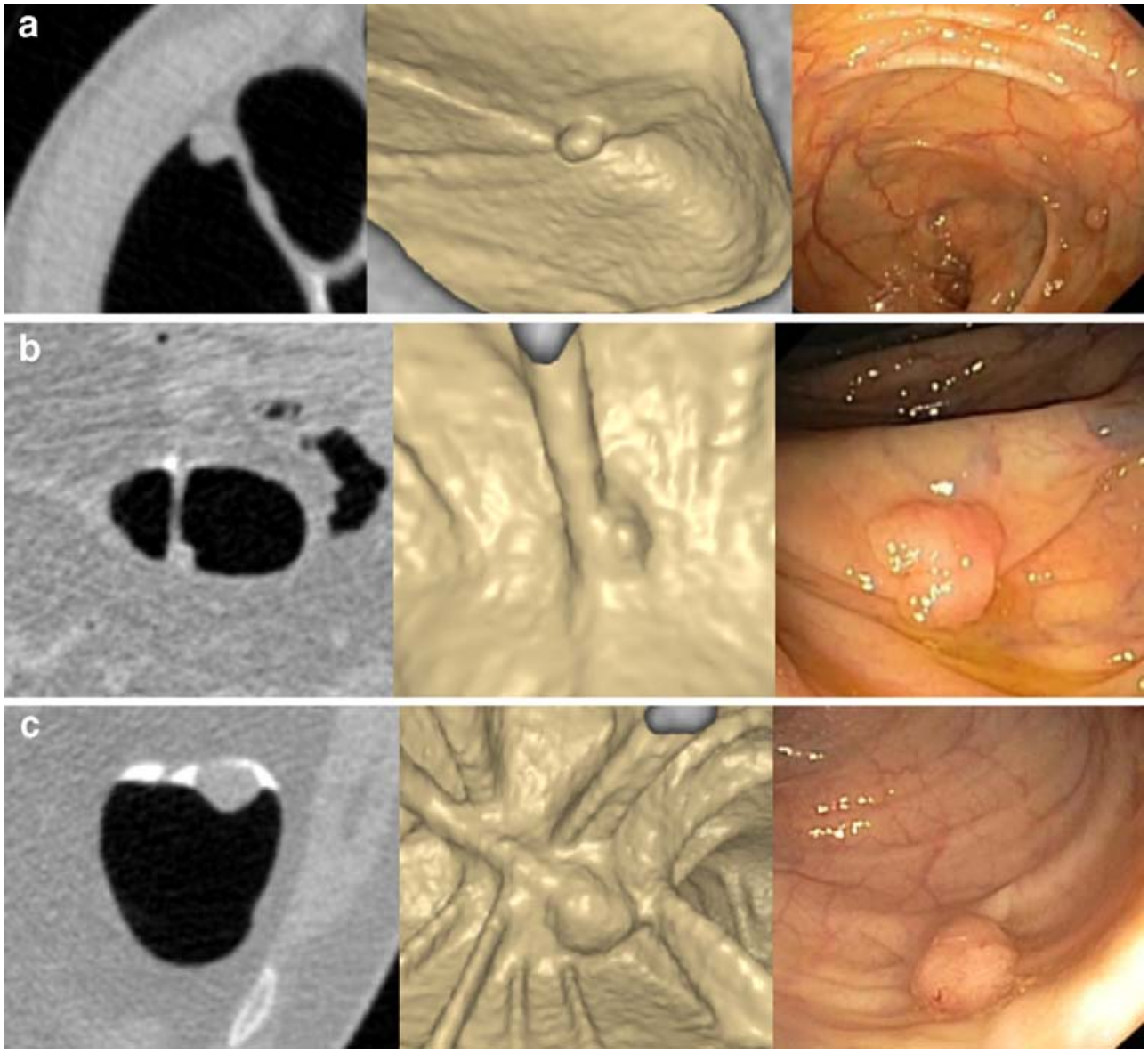

\section{Statistical analysis}

Because cases were preselected, only descriptive statistics were performed. A per case analysis was performed for the 21 single-polyp cases; for each case the number of the eight observers reporting a match was determined. For size and location matching we determined the number of instances in which a reader did not adhere to their own matching criteria, prespecified by them in the questionnaire.

Of the seven multiple-polyp cases, the number of matched polyps per size category $(\geq 10 \mathrm{~mm}, 6$ to $<10 \mathrm{~mm}$, or $<6 \mathrm{~mm}$ ) was counted per case and summarized per observer. Reference diameter was colonoscopic excepting nonmatched CTC polyps.

\section{Results}

Questionnaire

All readers stated that they normally use colonoscopy reports or a case record form completed during colonoscopy for polyp matching. Seven readers used video stills of colonoscopic polyps; four readers also used colonoscopy videos; two readers also employed pathology reports for polyp diameter information.

All readers normally required endoscopic information relating to segmental location, size, and morphology. Six readers defined a flat lesion as one whose width must be at least twice its height; two also stated that the polyp must protrude less than $3 \mathrm{~mm}$ from the mucosa. The remaining two readers exclusively used this latter definition for flat lesions. Three readers wished to have information about the distance of the polyp from the anus and two wished to have histology information to facilitate matching. Table 2 details the different criteria described by readers for matching.

\section{Single-polyp cases}

Agreement amongst readers could concern agreement on the presence of a match as well as the lack of presence of a match in a case, both important aspects in matching. Disagreement amongst readers in a case means that about half of the readers concern the presence of a match and the other half not. In the 21 cases with a single polyp, readers considered a match between CTC and OC to be present in between $13(62 \%)$ and $19(90 \%)$ of cases, i.e., there was 
Table 2 Different matching criteria indicated by eight readers in the questionnaire

\begin{tabular}{|c|c|c|}
\hline Morphology & Size & Location \\
\hline $\begin{array}{l}\text { CTC polyp needs to have a similar } \\
\text { appearance/shape as the colonoscopy } \\
\text { polyp ( } 8 \text { ) }\end{array}$ & $\begin{array}{l}\text { Size of the } C T C \text { polyp has to be within } 50 \% \text { of the } \\
\text { colonoscopy polyp size (4) }\end{array}$ & $\begin{array}{l}\text { Polyps are in the same or adjacent segment } \\
\text { (6) }\end{array}$ \\
\hline \multirow{3}{*}{$\begin{array}{l}\text { And description of morphology by the } \\
\text { gastroenterologist resembles that of } \\
\text { the CTC observer (6) }\end{array}$} & $\begin{array}{l}\text { Size of the } C T C \text { polyp can be maximally } 50 \% \text { smaller } \\
\text { or } 100 \% \text { larger than the colonoscopy polyp (1) }\end{array}$ & Polyps are in the same segment (1) \\
\hline & $\begin{array}{l}\text { Size of the colonoscopy polyp has to be within } 50 \% \text { (1) } \\
\text { or } 40 \% \text { (1) of the CTC polyp size }\end{array}$ & $\begin{array}{l}\text { Polyps are within a reasonable distance } \\
\text { (judged by the CTC radiologist who is } \\
\text { performing the matching) (1) }\end{array}$ \\
\hline & $\begin{array}{l}\text { Size of the CTC polyp has to be within } 50 \% \text { of the } \\
\text { colonoscopy polyp size or size of the colonoscopy } \\
\text { polyp has to be within } 50 \% \text { of the CTC polyp size (1) }\end{array}$ & \\
\hline
\end{tabular}

The numbers within parentheses indicate how many readers use the specific matching criterion

some disagreement as to whether a match between CTC and $\mathrm{OC}$ was possible or not due to a perceived unacceptable discrepancy for one or more of the typical matching criteria (size, location, and morphology).

To evaluate the magnitude of this disagreement we analyzed the per case agreement or disagreement. We then found that the readers agreed completely or almost completely in 15 of 21 cases with respect to the presence or lack of a match of CTC and colonoscopy findings. Complete agreement was present in five cases in whom all eight readers agreed on a match. Almost complete agreement on matching (i.e., seven of eight readers indicated a match) was present in seven cases, whereas almost complete agreement on the lack of matching was present in three cases. In six of the 21 cases, however, a considerable disagreement in matching was found. In five cases only four readers indicated a match, and in one case five readers indicated a match. Figure 2 indicates how many cases readers agreed and disagreed in matching the CTC and colonoscopy polyp.

To explore the rationale underpinning this disagreement we evaluated data separately for cases with location, size, and morphology discrepancies. In the five cases that were selected for segmental location difference between the CTC and colonoscopy polyp, there was a high matching agreement across readers. Nearly all readers refused to match polyps where the CTC and colonoscopy location differed by three or more adjacent segments.

Regarding the ten cases where diameter disagreements ostensibly prevented matching between the CTC and colonoscopy, in five cases nearly all readers in practice ignored diameter discrepancies of more than $100 \%$ between the CTC and colonoscopy polyp. In the other five cases, while diameter discrepancies of more than $100 \%$ again existed between polyps, only four of eight readers (in four cases) and five of eight readers (in one case) found matching possible, indicating poor agreement existed.
In cases with different morphology, agreement for matching was high except for a single case; case 21 demonstrated a fecal residue at CTC and a polyp at colonoscopy in which only four of eight readers performed a match between the fecal residue and a polyp.

Overall, 55 polyps (mean 6.9 polyps per reader) were matched by readers despite individual polyps not fulfilling the criteria for matching on the basis of diameter

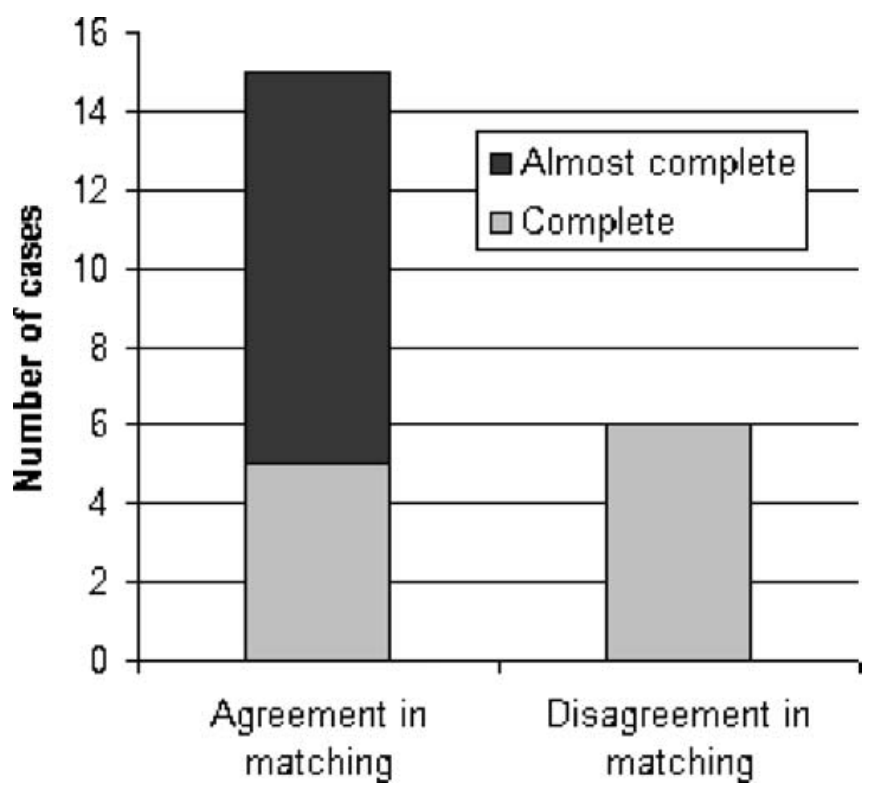

Fig. 2 Agreement and disagreement amongst readers in matching 21 single polyp cases. At the $x$-axis agreement or disagreement in matching is presented. The number of cases is given on the $y$-axis. In the ideal situation, all readers agree on the presence $(8 / 8)$ or the lack $(0 / 8)$ of a match; this means complete matching agreement (gray bar). When only half the readers agree on a match and the other half do not agree (4/8) this is complete matching disagreement (gray bar). When only seven of eight readers indicate a match or no match there is almost complete matching agreement (black bar) 
prespecified by each reader. Overall, 12 polyps (mean 1.5 polyps per reader) were matched despite not fulfilling criteria prespecified by readers for segmental location. Two polyps were matched despite not fulfilling both diameter and segmental criteria.

\section{Multiple-polyp cases}

The seven cases with multiple polyps had 11 CTC polyps and 12 colonoscopy polyps of $10 \mathrm{~mm}$ or larger, $18 \mathrm{CTC}$ and 12 colonoscopy polyps of 6-9 $\mathrm{mm}$, and nine CTC and 20 colonoscopy polyps smaller than $6 \mathrm{~mm}$. The total number of polyps matched per reader varied from 27 to 35 (Table 3). In case 3, for example, one reader matched five polyps on CTC with OC, while another reader matched nine. For CTC polyps of $10 \mathrm{~mm}$ or larger, the number of matches per reader showed less variation, between 9 and 11 (Table 4). For polyps smaller than $6 \mathrm{~mm}$ the inter-reader variability was larger, with the number of matches varied from 7 to 14 . The number of false negative CTC polyps of $10 \mathrm{~mm}$ or larger per reader ranged from one to three and the number of false positive CTC polyps of $10 \mathrm{~mm}$ or larger ranged from zero to two. Reasons for not matching CTC and colonoscopy polyps were mismatching due to location, size, and morphology.

\section{Observations}

Six readers remeasured polyps on CTC when a large diameter difference was apparent between it and the OC polyp. To resolve this, four readers disagreed with the diameter recorded by the colonoscopist and reinterpreted the size of the OC polyp shown on video. To determine polyp location precisely, two readers thoroughly examined the colonoscopic video to clarify the colonic segment or location of the polyp compared to a fold. Four readers occasionally scrutinized the morphology of the polyp at video, especially in pedunculated cases (e.g., to determine stalk length) and cases with flat polyps.

\section{Discussion}

We have investigated if disagreement exists between experienced readers when attempting to match polyps identified by CTC and subsequent colonoscopy. Eight experienced CTC researchers apparently used similar matching criteria, based on polyp location, size, and morphology. Readers largely agreed when matching cases with single polyps. We found, however, substantial disagreement in a minority but non-negligible proportion of cases. Disagreement was also present in cases with multiple polyps but predominantly for the least relevant polyps, i.e., those smaller than $6 \mathrm{~mm}$.

The CTC literature describes various matching criteria, based on expert opinion rather than an evidence-based approach. Evidence-based matching criteria are difficult to formulate because a robust reference standard for matching corresponding CTC and colonoscopy polyps poses very substantial methodological difficulties. We did not aim to validate matching criteria. Rather, we investigated how readers matched in practice and the level of disagreement between them.

While we found substantial agreement there was also non-negligible disagreement, predominantly due to a large perceived diameter difference between CTC and OC. This was not a constant observation, however, because some cases with similar discrepancies were matched by most readers. The reasons underpinning this observation were unclear, despite us asking readers for their rationale.

Discrepancy was also noted in those cases with multiple polyps. For the most clinically important polyps ( $\geq 10 \mathrm{~mm}$ ), we observed minimal disagreement. In populations with a low prevalence of polyps, matching is less problematic because few polyps need be matched [6]. However, when the number of polyps per patient increases, matching will

Table 3 Number of matched polyps per reader in the multiple-polyp cases

\begin{tabular}{|c|c|c|c|c|c|c|c|c|}
\hline & \multicolumn{8}{|c|}{ Reader } \\
\hline & 1 & 2 & 3 & 4 & 5 & 6 & 7 & 8 \\
\hline Case 1 & 10 & 9 & 10 & 9 & 10 & 8 & 10 & 11 \\
\hline Case 2 & 9 & 9 & 9 & 8 & 8 & 7 & 9 & 9 \\
\hline Case 3 & 7 & 8 & 8 & 8 & 8 & 5 & 7 & 9 \\
\hline Case 4 & 0 & 0 & 0 & 0 & 1 & 1 & 1 & 0 \\
\hline Case 5 & 2 & 4 & 3 & 3 & 3 & 3 & 2 & 3 \\
\hline Case 6 & 2 & 2 & 2 & 2 & 2 & 2 & 2 & 2 \\
\hline Case 7 & 1 & 2 & 1 & 1 & 1 & 1 & 1 & 1 \\
\hline Total & 31 & 34 & 33 & 31 & 33 & 27 & 32 & 35 \\
\hline
\end{tabular}

The numbers of matched polyps (all sizes) are indicated per reader. The total number of matches per reader is presented in the last row 
Table 4 Numbers of true positive, false positive, and false negative CTC polyps per size category per reader in the multiple-polyp cases

\begin{tabular}{|c|c|c|c|c|c|c|c|c|}
\hline & \multicolumn{8}{|c|}{ Reader } \\
\hline & 1 & $2^{a}$ & 3 & 4 & 5 & 6 & 7 & 8 \\
\hline $\mathrm{TP} \geq 10 \mathrm{~mm}$ & 11 & 11 & 11 & 10 & 9 & 10 & 11 & 10 \\
\hline TP 6-9 mm & 10 & 10 & 9 & 10 & 11 & 10 & 8 & 11 \\
\hline $\mathrm{TP}<6 \mathrm{~mm}$ & 10 & 13 & 13 & 11 & 13 & 7 & 13 & 14 \\
\hline $\mathrm{FN} \geq 10 \mathrm{~mm}$ & 1 & 1 & 1 & 2 & 3 & 2 & 1 & 2 \\
\hline FN 6-9 mm & 2 & 2 & 3 & 2 & 1 & 2 & 4 & 1 \\
\hline $\mathrm{FN}<6 \mathrm{~mm}$ & 10 & 6 & 7 & 9 & 7 & 13 & 7 & 6 \\
\hline $\mathrm{FP} \geq 10 \mathrm{~mm}$ & 0 & 0 & 0 & 1 & 1 & 2 & 0 & 0 \\
\hline FP 6-9 mm & 2 & 1 & 1 & 2 & 0 & 6 & 2 & 1 \\
\hline $\mathrm{FP}<6 \mathrm{~mm}$ & 5 & 0 & 3 & 3 & 4 & 3 & 4 & 4 \\
\hline
\end{tabular}

$T P$ true positive polyp, i.e., a CTC polyp that was matched with a colonoscopy polyp; $F N$ false negative polyp for CTC, i.e., a colonoscopy polyp that was not matched with a CTC polyp; FP false positive polyp for CTC, i.e., a CTC polyp that was not matched with a colonoscopy polyp

${ }^{\mathrm{a}}$ Reader 2 had interpreted one case wrongly and this case was therefore excluded

likely become less straightforward and we have demonstrated inter-reader disagreement.

After case matching, readers were asked for their matching criteria via a questionnaire. All readers reported practically identical matching criteria (described in the Introduction). While these criteria are apparently straightforward, in practice there are several problems. Difficulties when matching location exist because anatomical borders are ill-defined and colonoscopists frequently cannot locate the endoscope tip with precision [9]. In our study almost all readers took this into account and were prepared to match polyps that were not within the same or adjacent colonic segments.

Another problem exists when matching based on polyp diameter. Colonoscopic estimation of diameter is imprecise $[10,11]$. CTC measurements are also variable but are probably more accurate [12-14]. We found that most readers remeasured CTC polyps and often redefined the colonoscopist's assessment of diameter from the video provided. Hence apparently large diameter differences between polyps did not always preclude a match.

Matching of polyps based on morphology also differed between readers because judgment of morphology is subjective. While definitions of lesion morphology are clearly described [15], we found that readers often used different definitions for flat lesions at CTC.

Because we found that experienced readers did not always adhere to established matching criteria, we propose that disagreement is best resolved by consensus. At the very least, two readers would then have to consider whether a match between a polyp imaged by both CTC and OC was possible, which is likely to reduce error and uncertainty. Such an approach is inevitably time consuming and an alternative is to perform consensus matching only when polyps do not satisfy generally accepted criteria for matching. However, as we have stated, these criteria are not evidence-based and our study was not designed to provide such a base. However, we do propose a matching procedure (Fig. 3) suggesting consensus matching by at least two experienced readers where cases do not satisfy conventional matching criteria. Optimally, at least one observer should be a radiologist and the other a colonoscopist since both have different attributes.

A potential limitation of our study is that we did not present pathology reports or the histological diameter of excised polyps. It is however questionable whether these data would provide useful additional information since polyps often shrink after polypectomy due to electrosurgical tissue effects and vascular collapse [16]. Another limitation is that our cases were purposely biased towards difficult cases. This was done to magnify any inter-reader variation in a pragmatic manner. Although this approach was efficient, as a consequence it was impossible to calculate meaningful metrics applicable to real-world scenarios.

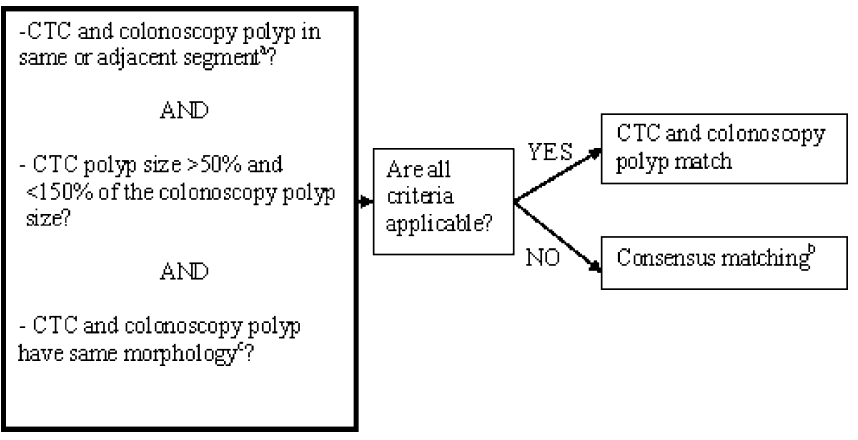

Fig. 3 Matching procedure of CTC and colonoscopy polyps. a Six colonic segments are considered: caecum, ascending, transverse and descending colon, sigmoid, and rectum. b Consensus matching must be performed with at least two experienced persons, preferably one radiologist and one gastroenterologist. c CTC and colonoscopy polyp have a similar appearance/shape (judged by the observer who is performing the matching) 
In summary, we found that experienced CTC readers agree to a considerable extent when matching polyps detected by CTC to those found at subsequent OC in difficult cases, but non-negligible disagreement exists. Such disagreement may explain data variation of some studies on the diagnostic accuracy of CTC. We suggest using a consensus to minimize disagreement when match- ing those cases that do not satisfy established matching criteria.

Open Access This article is distributed under the terms of the Creative Commons Attribution Noncommercial License which permits any noncommercial use, distribution, and reproduction in any medium, provided the original author(s) and source are credited.

\section{References}

1. Levin B, Lieberman DA, McFarland B et al (2008) Screening and surveillance for the early detection of colorectal cancer and adenomatous polyps, 2008: a joint guideline from the American Cancer Society, the US Multi-Society Task Force on Colorectal Cancer, and the American College of Radiology. Gastroenterology 134(5):1570-1595

2. Dachman AH, Zalis ME (2004) Quality and consistency in CT colonography and research reporting. Radiology 230 (2):319-323

3. Zalis ME, Barish MA, Choi JR et al (2005) CT colonography reporting and data system: a consensus proposal. Radiology 236(1):3-9

4. Iannaccone $\mathrm{R}$, Laghi $\mathrm{A}$, Catalano $\mathrm{C}$ et al (2004) Computed tomographic colonography without cathartic preparation for the detection of colorectal polyps. Gastroenterology 127(5):13001311

5. Johnson CD, Toledano AY, Herman BA et al (2003) Computerized tomographic colonography: performance evaluation in a retrospective multicenter setting. Gastroenterology 125 (3):688-695
6. Pickhardt PJ, Choi JR, Hwang I et al (2003) Computed tomographic virtual colonoscopy to screen for colorectal neoplasia in asymptomatic adults. $\mathrm{N}$ Engl J Med 349(23):2191-2200

7. Taylor SA, Charman SC, Lefere P et al (2008) CT colonography: investigation of the optimum reader paradigm by using computer-aided detection software. Radiology 246(2):463-471

8. van Gelder RE, Nio CY, Florie J et al (2004) Computed tomographic colonography compared with colonoscopy in patients at increased risk for colorectal cancer. Gastroenterology 127(1):41-48

9. Shah SG, Saunders BP, Brooker JC, Williams CB (2000) Magnetic imaging of colonoscopy: an audit of looping, accuracy and ancillary maneuvers. Gastrointest Endosc 52(1):1-8

10. Punwani $S$, Halligan $S$, Irving $P$ et al (2008) Measurement of colonic polyps by radiologists and endoscopists: Who is most accurate? Eur Radiol 18 (5):874-881

11. Gupta S, Durkalski V, Cotton P, Rockey DC (2008) Variation of agreement in polyp size measurement between computed tomographic colonography and pathology assessment: clinical implications. Clin Gastroenterol Hepatol 6 (2):220-227
12. Burling D, Halligan S, Taylor S et al (2006) Polyp measurement using CT colonography: agreement with colonoscopy and effect of viewing conditions on interobserver and intraobserver agreement. AJR Am J Roentgenol 186 (6):1597-1604

13. Park SH, Choi EK, Lee SS et al (2007) Polyp measurement reliability, accuracy, and discrepancy: optical colonoscopy versus CT colonography with pig colonic specimens. Radiology 244 (1):157-164

14. Taylor S, Slater A, Honeyfield L, Burling D, Halligan S (2006) CT colonography: effect of colonic distension on polyp measurement accuracy and agreement-in vitro study. Acad Radiol 13(7):850-859

15. Participants in the Paris workshop (2003) he Paris endoscopic classification of superficial neoplastic lesions: esophagus, stomach, and colon: November 30 to December 1, 2002. Gastrointest Endosc 58(6 Suppl):S3-S43

16. Gopalswamy N, Shenoy VN, Choudhry U et al (1997) Is in vivo measurement of size of polyps during colonoscopy accurate? Gastrointest Endosc 46(6):497-502 